\title{
Modified arytenoid muscle electrode recording method for neuromonitoring during thyroidectomy
}

\author{
Peng Li, Qing-Zhuang Liang, Dong-Lai Wang, Bin Han, Xin Yi, Wei Wei \\ Department of Thyroid and Parathyroid Surgery, Peking University Shenzhen Hospital, Peking University Health Science Center, Shenzhen \\ 518000, China \\ Contributions: (I) Conception and design: P Li; (II) Administrative support: W Wei; (III) Provision of study materials or patients: QZ Liang, DL \\ Wang; (IV) Collection and assembly of data: B Han; (V) Data analysis and interpretation: X Yi; (VI) Manuscript writing: All authors; (VII) Final \\ approval of manuscript: All authors. \\ Correspondence to: Wei Wei, MD. Department of Thyroid and Parathyroid Surgery, Peking University Shenzhen Hospital, 1120 Lianhua Road, \\ Futian District, Shenzhen 518000, China. Email: rxwei1123@163.com.
}

Background: Intraoperative neuromonitoring (IONM) is an important application for protecting recurrent laryngeal nerve (RLN) during thyroid surgery. The method for recording arytenoid muscle electromyography (EMG) signals is reported to be feasible and reliable. However, the parameters of EMG signals are not provided. This study aimed to analyze the clinical characteristics of EMG signal parameters by modifying the insertion direction of needle electrodes.

Methods: A total of 92 patients who were scheduled to undergo thyroidectomy were recruited. Two paired needle electrodes were inserted in bilateral angle points between rectus cricothyroid muscle and inferior margin of thyroid cartilage (TC) intraoperatively, and then the information from the EMG signals was recorded according to four-step method (V1-R1-R2-V2). Pre-and post-operative laryngo-fiberoscopy was performed to confirm the vocal cord function.

Results: A total of 122 RLNs were successfully recorded during thyroidectomy, with the mean EMG amplitude and latency were $1,857 \pm 1,718 / 2,347 \pm 2,323 \mu \mathrm{V}$ and $3.89 \pm 1.12 / 2.26 \pm 0.05 \mathrm{~ms}$ for $\mathrm{V} 1 / \mathrm{R} 1$ signals before resection, and $1,924 \pm 1,705 / 2,450 \pm 2,345 \mu \mathrm{V}$ and $3.87 \pm 1.17 / 2.27 \pm 0.08 \mathrm{~ms}$ for R2/V2 signals after resection. There were no significant changes before and after resection, and a normal vocal cord movement was observed postoperatively. The amplitude of left nerves was higher than that of the right ones. Furthermore, the latency of the right vagus was shorter than the left ones, but there was no difference in the amplitude and latency between age, sex and pathological types.

Conclusions: Modified arytenoid muscle EMG recording method was considered to be safe, feasible and reliable. The latency of right vagus EMG signals were shorter than the left ones, and the amplitude of EMG signals might be related to different sides.

Keywords: Arytenoid muscle; electromyography signals; intraoperative neuromonitoring (IONM); recurrent laryngeal nerve (RLN); thyroidectomy

Submitted May 18, 2019. Accepted for publication Aug 14, 2019.

doi: $10.21037 /$ gs.2019.08.07

View this article at: http://dx.doi.org/10.21037/gs.2019.08.07

\section{Introduction}

With the advancements of anatomical knowledge and surgical techniques, the incidence of recurrent laryngeal nerve (RLN) injury has been dramatically reduced, but the temporary and permanent RLN injuries still accounted for approximately $9.8 \%$ and $2.3 \%$ (1). Unilateral RLN injury resulted in hoarseness, while bilateral RLN injuries led to airway compromise, requiring emergent tracheostomy. Furthermore, it has been reported to be one of the most 
common causes of medicolegal litigation (2). In recent decades, intraoperative neuromonitoring (IONM) is widely used in clinics as an important assistant tool for protecting RLN (3). Moreover, the International Neural Monitoring Study Group (INMSG) have published international standard guidelines for standardizing the IONM procedure (4).

In 1969, Flisberg et al. have first reported transcricothyroid electromyography signal (EMG) recording method for IONM of RLN during thyroidectomy (5). In recent years, Petro (6) and Alon (7) have separately inserted two and single paired needle electrodes into the arytenoid muscle for IONM during thyroid surgery. These methods are reported to be feasible and reliable, but the parameters of EMG signals are not provided. Hence, in this study, the clinical characteristics of the parameters of EMG were analyzed by modifying the insertion direction of needle electrodes.

\section{Methods}

\section{Criteria for admission of patients}

From May 2017 to September 2018, patients with thyroid disease who were admitted to the Department of Thyroid Surgery (Peking University Shenzhen Hospital, Shenzhen, China) were enrolled in this study. The inclusion criteria were as follows: (I) patients with early differentiated thyroid cancer (DTC); (II) patients with benign thyroid disease, with a maximum diameter of more than $4 \mathrm{~cm}$ or symptoms of compression; and (III) primary hyperthyroidism patients scheduled for surgical treatment. The exclusion criteria were as follows: patients (I) with vocal cord paralysis as indicated by preoperative electronic laryngoscope; (II) with locally advanced DTC; (III) with undifferentiated thyroid cancer; and (IV) who underwent a redo thyroidectomy. Written informed consent was obtained from all patients, and they were informed regarding the intent to use IONM system that potentially aids in the localization and identification of RLNs, as well as to assess their function during operation. This study was approved by the Ethics Committee of Peking University Shenzhen Hospital (2019-024).

\section{Evaluation of vocal cord function before and after operation}

All patients underwent pre- and post-operative laryngofiberoscopic examination of the vocal cord function. If there is vocal cord paralysis, the electronic laryngoscope was performed again at 1, 3 and 6 months after operation. If the vocal cord function has not been recovered for more than 6 months, then it was regarded as permanent vocal cord paralysis. The above information was recorded in detailed.

\section{IONM setup and surgical procedure}

All patients underwent general anesthesia induced with lidocaine $(1 \mathrm{mg} / \mathrm{kg})$, propofol $(4.0 \mu \mathrm{g} / \mathrm{L})$, sufentanil $(0.5 \mu \mathrm{g} / \mathrm{kg})$, rocuronium bromide $(0.3 \mathrm{mg} / \mathrm{kg})$, and targetcontrolled infusion (TCI). After performing assisted ventilation for $5 \mathrm{~min}$, the standard endotracheal tubes (male: $7 \mathrm{~mm}$, female: $6 \mathrm{~mm}$; Medtronic Xomed Inc., FL, USA) were inserted by assisted visible laryngoscope. Anesthesia maintenance was administered as follows: propofol $(3.5-4.0 \mu \mathrm{g} / \mathrm{L})$ as TCI continuous intravenous pumping and remifentanil $(0.1 \mu \mathrm{g} / \mathrm{kg} / \mathrm{min})$ as continuous intravenous pumping.

The operation was performed by the same surgical team (Dr. P Li and QZ Liang). After successful anesthetic intubation, shoulders were cushioned with pillows to achieve cervical hyperextension. Standard procedures for thyroidectomy are implemented, followed by observation and palpation for the identification of bilateral angle points between the rectus cricothyroid muscle and the inferior margin of thyroid cartilage (TC). The two paired needle electrodes (length, $12.0 \mathrm{~mm}$; diameter, $0.4 \mathrm{~mm}$; Medtronic Xomed Inc., FL, USA) were then inserted in an outward and upward manner from the bilateral angle points to the arytenoid muscle (Figure 1A,B), The average time of needle electrodes placement is about $10 \mathrm{~s}$. The channel leads from the needle electrodes were connected to the NIM-Response 3.0 (Medtronic Xomed Inc., FL, USA) Patient Interface. Head and neck procedure was selected to enter the thyroid mode. The monitoring system generates stimuli with a time window set to $50 \mathrm{~ms}$ and an amplitude scale set to $0.2 \mathrm{mV} /$ division. The pulsed stimuli were $100 \mu$ s in duration and $4 \mathrm{~Hz}$ in frequency. The capturing of events was activated at a threshold of $100 \mu \mathrm{V}$. A standardized IONM procedure $\left(V_{1}-R_{1}-R_{2}-V_{2}\right.$ signals) was strictly followed, and RLN was routinely mapped and exposed.

\section{Data recording and processing methods}

Microsoft Office Excel 2007 was used for recording the data related to sex and age of the patients, and the results of laryngo-fiberoscopic examinations, and pathology. Moreover, the amplitude and latency of EMG before and after resection were expressed as mean \pm standard deviation. 

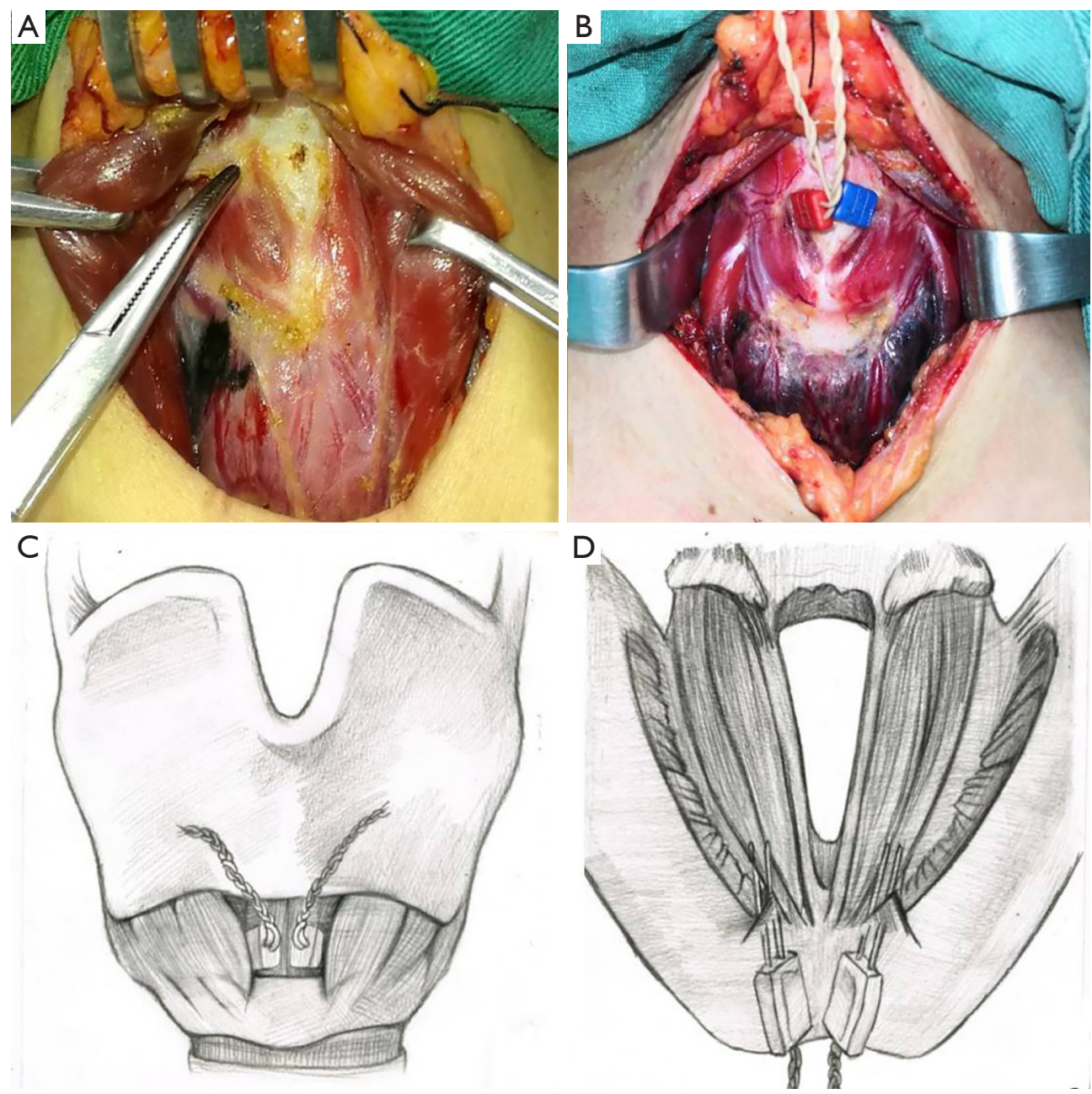

Figure 1 The modified arytenoid muscle EMG recording method: (A) visual identification of bilateral angle points between rectus cricothyroid muscle and inferior margin of TC; (B) two paired needle electrodes were outwards and upwards inserted into the bilateral angle points to arytenoid muscle; (C) needle electrodes were inserted outwards and upwards to avoid insertion into the cricoid cartilage and cricothyroid muscle; (D) the needle electrode were inserted into the superior arytenoid muscle and far from the vocal cord. EMG, electromyography; TC, thyroid cartilage.

GraphPad Prism 7.0 software was used to compare the differences among all parameters before and after surgery. A $\mathrm{P}$ value of $\leq 0.05$ was considered to be statistically significant.

\section{Results}

A total of 92 patients including 35 men and 57 women, with a mean age of 40 years (range, 23-66 years) have successfully undergone thyroidectomy. There were 76 cases with papillary thyroid cancer (PTC), 8 cases with nodular goiter, 6 cases with follicular adenoma and 2 cases with primary hyperthyroidism. All the 124 nerves at risk (NAR) were recorded for typical EMG signals. The two RLN signals were lost during the operation. On the next day, electronic fiberoptic laryngoscope showed that one case had normal vocal cord movement, and another case with vocal cord dyskinesia was followed up for 6 months without recovery, and the remaining 90 cases (122 NAR) showed normal vocal cord movement. Two cases had a small amount of bleeding at the needling point, but achieved hemostasis after pressing for 5 minutes. No local hematoma, infection or broken needle electrode occurred in all cases.

In 122 NARs, the mean EMG amplitude was 
Table 1 Clinical characteristics of the amplitude for 122 NARs

\begin{tabular}{|c|c|c|c|c|c|}
\hline Variables & NAR (n) & \multicolumn{4}{|c|}{ Amplitude } \\
\hline Sex & & & 0.9640 & & 0.9043 \\
\hline Male & 40 & $1,867 \pm 1,802$ & & $2,384 \pm 2,285$ & \\
\hline Female & 82 & $1,852 \pm 1,676$ & & $2,330 \pm 2,341$ & \\
\hline$\leq 40$ & 71 & $1,832 \pm 1,539$ & & $2,225 \pm 1,866$ & \\
\hline$>40$ & 51 & $1,890 \pm 1,940$ & & $2,518 \pm 2,831$ & \\
\hline Pathological types & & & 0.6284 & & 0.5394 \\
\hline Benign diseases & 20 & $2,027 \pm 1,302$ & & $2,640 \pm 1,219$ & \\
\hline Left & 51 & $2,233 \pm 2,213$ & & $2,874 \pm 3,003$ & \\
\hline Right & 71 & $1,586 \pm 1,175$ & & $1,969 \pm 1,566$ & \\
\hline
\end{tabular}

*, statistically significant. NAR, nerves at risk; V1, amplitude of vagus; R1, amplitude of RLN; RLN, recurrent laryngeal nerve.

$1,857 \pm 1,718 / 2,347 \pm 2,323 \mu \mathrm{V}$ for $\mathrm{V} 1 / \mathrm{R} 1$ signals before resection, and $1,924 \pm 1,705 / 2,450 \pm 2,345 \mu \mathrm{V}$ for $\mathrm{R} 2 /$ V2 signals after resection. No significant changes in EMG signals before and after resection $(\mathrm{P}=0.7601$ and $0.7306)$ were observed. The mean EMG latency was $3.89 \pm 1.12 / 2.26 \pm 0.05 \mathrm{~ms}$ for $\mathrm{V} 1 / \mathrm{R} 1$ signals before resection, and $3.87 \pm 1.17 / 2.27 \pm 0.08 \mathrm{~ms}$ for $\mathrm{R} 2 / \mathrm{V} 2$ signals after resection. There were no significant differences before and after resection $(\mathrm{P}=0.8916$ and 0.2428$)$.

The clinical characteristics of EMG parameters were analyzed. The amplitude of left and right V1/R1 were $2,233 \pm 2,213 / 2,874 \pm 3,003 \mu \mathrm{V}$ and $1,586 \pm 1,175 / 1,969 \pm 1,566$ $\mu \mathrm{V}$. This showed that the amplitude of left vagus and RLN were higher than the right ones $(\mathrm{P}=0.0388$ and 0.0093$)$. The latency of left and right vagus nerves was $5.02 \pm 0.70$ and $3.08 \pm 0.50 \mathrm{~ms}$, the latency of right vagus was shorter than the left ones $(\mathrm{P}<0.0001)$, but showed no significant difference in the amplitude and latency between different ages, sex and pathological types (Tables 1,2).

\section{Discussion}

Visual identification is considered as the gold standard for RLN protection during thyroid surgery. As an assistant tool, IONM has been accepted and used by several surgeons. According to the survey of American Association of Endocrine Surgeons (AAES), there is an increase in the use of IONM from $7 \%$ in 2001 to $37 \%$ in 2007 (8). The tracheal tube electrode for IONM during thyroid surgery was first reported in 1996 (9). Because of the essential advantages of easy setup and use, noninvasive nature, and capacity to derive larger areas of the target muscle, it is widely used in thyroid surgery. But there are still some limitations, which were as follows: (I) verifying the proper electrodes-cords position and readjusting the EMG tube remains troublesome and time-consuming for anesthesiologists; (II) displacement of EMG tube during surgical maneuvers on thyroid lobe or trachea often occurs, and may cause unstable EMG signals; (III) replacement of EMG tube is difficult when IONM is unexpectedly required during the operation; and (IV) the cost of EMG tube is relatively high (10-12).

The history of arytenoid muscle electrode for IONM during thyroidectomy was much early than that of tracheal tube electrode, and this was first reported by Flisberg et al. in 1969 (5). In recent years, Petro (6) and Alon (7) have separately inserted the two and single paired needle electrodes into the arytenoid muscle and IONM was performed during thyroid surgery. However, the insertion locations are both located in the central area of the cricothyroid membrane, where penetration into the vocal cord might not be completely avoided, and so we changed 
Table 2 Clinical characteristics of latency for 122 NARs

\begin{tabular}{|c|c|c|c|c|c|}
\hline Variables & NAR (n) & \multicolumn{4}{|c|}{ Latency } \\
\hline Sex & & & 0.8545 & & $>0.9999$ \\
\hline Male & 40 & $3.92 \pm 1.25$ & & $2.26 \pm 0.04$ & \\
\hline Female & 82 & $3.88 \pm 1.06$ & & $2.26 \pm 0.06$ & \\
\hline$\leq 40$ & 71 & $3.82 \pm 1.11$ & & $2.26 \pm 0.03$ & \\
\hline$>40$ & 51 & $4.00 \pm 1.13$ & & $2.26 \pm 0.07$ & \\
\hline Pathological types & & & 0.4672 & & $>0.9999$ \\
\hline Benign diseases & 20 & $4.06 \pm 1.18$ & & $2.26 \pm 0.03$ & \\
\hline Left & 51 & $5.02 \pm 0.70$ & & 2. $26 \pm 0.04$ & \\
\hline Right & 71 & $3.08 \pm 0.50$ & & $2.26 \pm 0.06$ & $>0.9999$ \\
\hline
\end{tabular}

*, statistically significant. NAR, nerves at risk; $V 1$, latency of vagus; R1, latency of RLN; RLN, recurrent laryngeal nerve.

the insertion needle direction to the superior of arytenoid muscle and far from vocal cord. We believed that this modification had the following advantages: (I) the insertion position of needle electrodes has visual anatomical landmark and can easily locate during the operation; (II) insertion of needle tip into the superior of arytenoid muscle avoids injury to the vocal cord; (III) the obtained EMG signal is strong enough to meet the needs of IONM; and (IV) the needle electrodes are not located in the central area, but have little influence on the operation (Figure 1C,D).

Various EMG recording methods for IONM during thyroid surgery have been reported in the literature. $\mathrm{Wu}$ et al. have first reported the feasibility of transcutaneous EMG recording method in an animal study (13). However, the low EMG amplitudes recorded by this method are unreliable and limited in clinical practice. An alternative trans-TC EMG recording method that is feasible, safe, and reliable has been put forwarded by conducting several clinical and animal studies $(14,15)$, but it is affected by the hardness of TC. The surface electrodes of TC obtained had relatively strong EMG signals and showed no invasiveness, but additional free TC is needed during the operation (16,17). In this study, the mean EMG amplitude of EMG signals were $1,857 \pm 1,725 / 2,347 \pm 2,333 \mu \mathrm{V}$ and the maximum amplitude can be greater than $7,000 \mu \mathrm{V}$ for vagus and RLNs (Figure 2A,B). Moreover, it was unaffected by the hardness of TC and did not require additional free TC, and the time-consuming of needle electrodes placement is about $10 \mathrm{~s}$. So, we believed that this method was clinically feasible and convenient.

The arytenoid muscle EMG signal recording method is an invasive procedure, and so the direction of needle insertion was modified. The paired needle electrodes do not penetrate the cricothyroid muscle and vocal cord, and so the incidence of muscle hematoma was rather low. But there may be small blood vessels between the cricothyroid muscle and TC, and we tried to avoid puncturing of these small blood vessels as far as possible. In this study, 2 cases had bleeding at the insertion point, and hemostasis was achieved by pressing for a short time. In order to avoid cricothyroid muscle injury, energy devices are not recommended for hemostasis.

This study suggested that the latency of left vagus was longer than those of the right. This was consistent with the results reported in Phelan et al. and Lorenz et al. study $(18,19)$, and the possible reason for this is due to longer left conduction pathway. Interestingly, this study also showed that the amplitudes of left vagus and RLN EMG signals were higher than those of the right. We speculated that this may be due to the fact that our surgical team was always accustomed to perform thyroidectomy in the right side first. When left side thyroidectomy was initiated, the EMG 


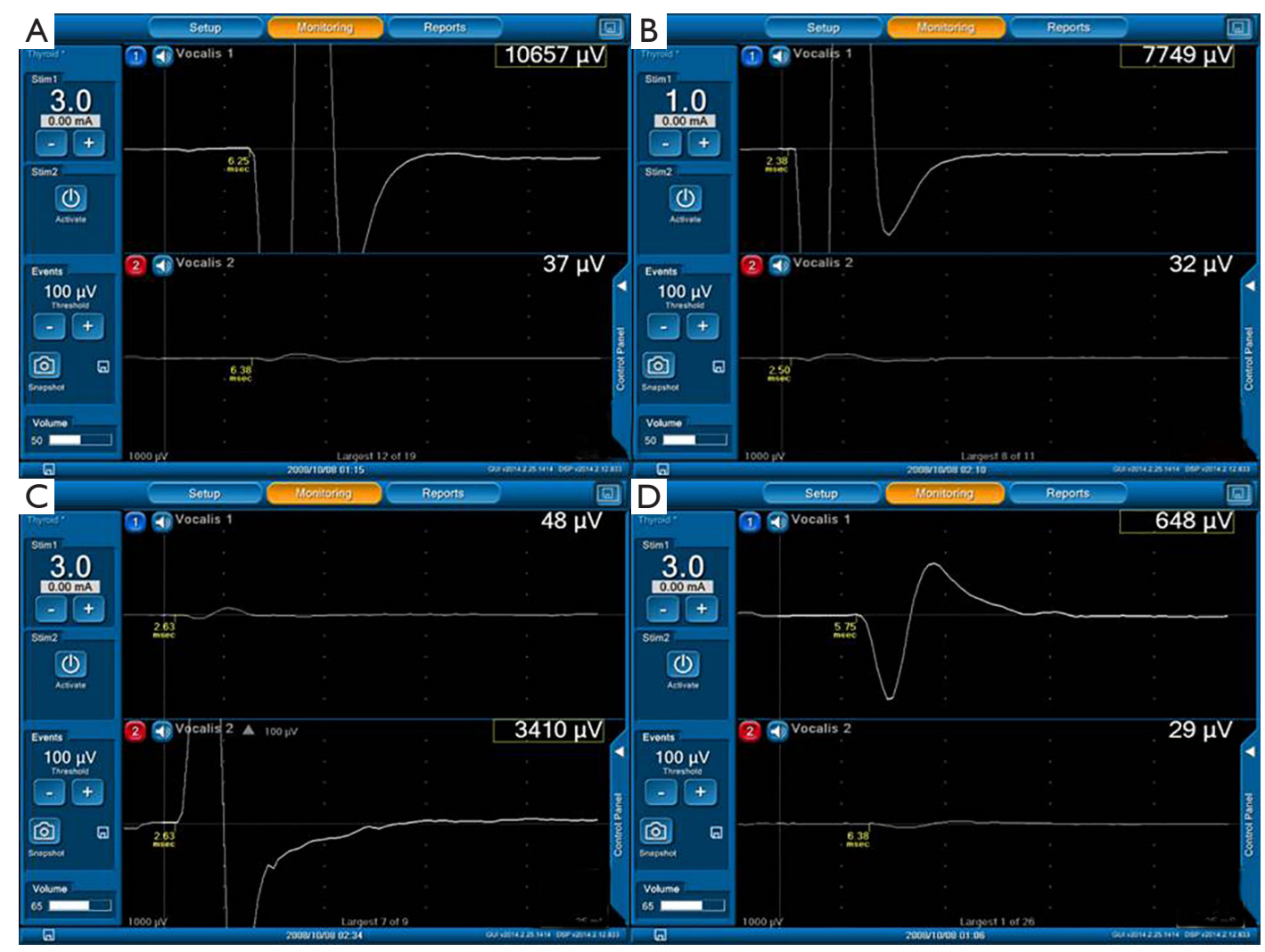

Figure 2 The EMG signals of modified arytenoid muscle recording method: (A) the maximum amplitude of vagus nerve. It is a typical three-directional waveform with a latency of $6.25 \mathrm{~ms}$. When the stimulating current is $3 \mathrm{~mA}$, the amplitude can be as high as $10,657 \mu \mathrm{V}$; (B) the maximum amplitude of RLN, and is also three-directional waveform with a latency of $6.25 \mathrm{~ms}$. When the stimulating current is $1 \mathrm{~mA}$, the amplitude can be as high as $7,749 \mu \mathrm{V} ;(\mathrm{C})$ the right needle electrode was placed in the right direction, and when the stimulating current was $3 \mathrm{~mA}$, the amplitude of vagus nerve EMG signal was 3,410 $\mu \mathrm{V}$; (D) when the left needle electrodes of the same patient were placed into the TC, the amplitude of vagus nerve EMG signal was only $648 \mu \mathrm{V}$. EMG, electromyography; RLN, recurrent laryngeal nerve.

signals may become stronger to complete due to metabolism muscle relaxants. In addition, we found that if the insertion direction is inappropriate, the amplitude of EMG signals on both sides in the same patient may show obvious differences (Figure 2C,D). According to our experience, the insertion direction should be gently modified either upward or outward obliquely through the spaces between cricothyroid muscle and TC. If the resistance was high, then the direction should be adjusted in time to avoid penetration into the TC.

In addition, we found that the amplitudes of EMG signals recorded by modified transcricothyroid method were obvious differences $(1,857 \pm 1,718 / 2,347 \pm 2,323 \mu \mathrm{V}$ for $\mathrm{V} 1$ / $\mathrm{R} 1$ ), even between the left and right sides of same patient, We speculated that it may be related to the direction of needle electrode implantation (Figure $2 C, D$ ). Storck et al. (20) had studied cadaveric specimens by 3-dimensional magnetic resonance tomography, he found that when the needle was inserted from the center of inferior border of TC, if the target muscle is the thyroarytenoid muscle (TAM), the needle has to be deflected laterally by $30^{\circ}$ and upward by $15^{\circ}$, and if we want to reach the lateral cricoarytenoid muscle (LCAM), the needle has to be deflected laterally by $30^{\circ}$ and downward by less than $15^{\circ}$. The insertion depth is about $15 \mathrm{~mm}$ for the TAM and 20 $\mathrm{mm}$ for the LCAM. In this study, our target muscle was arytenoid muscle, so we also followed the puncture method by Storck et al. However, due to the individual differences in laryngeal size (especially between men and women) and the length of needle electrode (only $12 \mathrm{~mm}$ ), the end of the needle electrode may not reach the arytenoid muscle in some cases. Instead, it reached the fat layer between TAM 
and LCAM, even within the LCAM.

The amplitudes of EMG signals for 122 NARs showed no significant changes before and after resection. The normal vocal cord movement was confirmed by electronic fibrolaryngoscope after operation. The negative predictive value (NPV) was $100 \%$, and this was similar to that of the electrode of tracheal intubation $(21,22)$. Two RLN EMG signals were lost, but the electronic fiberoptic laryngoscope showed that one case had normal vocal cord movement and in another case had vocal cord dyskinesia. However, the positive predictive value (PPV) should be further validated by clinical trials with larger sample size.

However, our study has some limitations, which were as follows: (I) the number of cases is rather small, and required animal experiments and larger clinical trials to verify its safety and validity; (II) there are fewer positive events, and its PPV should be further verified by larger clinical trials; and (III) this study lacked control group, and with this more cases can be monitored simultaneously by tracheal intubation vocal cord surface electrode monitoring method.

\section{Conclusions}

In conclusion, the arytenoid muscle electrode recording method proposed in this study was considered to be safe, feasible and reliable. The latency of right vagus EMG signals were shorter than the left ones and the amplitude of EMG signals might be related to the differences in the sides, but not related to age, sex, and pathological types of thyroid disease.

\section{Acknowledgments}

Funding: This work was supported by Shenzhen San-Ming Project (Grant No. SZSM201612010).

\section{Footnote}

Conflicts of Interest: The authors have no conflicts of Interest to declare.

Ethical Statement: The authors are accountable for all aspects of the work in ensuring that questions related to the accuracy or integrity of any part of the work are appropriately investigated and resolved. This study was approved by the Ethics Committee of Peking University Shenzhen Hospital (2019-024). Participant gave informed consent before taking part to this study.

\section{References}

1. Jeannon JP, Orabi AA, Bruch GA, et al. Diagnosis of recurrent laryngeal nerve palsy after thyroidectomy: a systematic review. Int J Clin Pract 2009;63:624-9.

2. Abadin SS, Kaplan EL, Angelos P. Malpractice litigation after thyroid surgery: the role of recurrent laryngeal nerve injuries, 1989-2009. Surgery 2010;148:718-22; discussion 722-3.

3. Yang $\mathrm{S}, \mathrm{Zhou} \mathrm{L}, \mathrm{Lu} \mathrm{Z}$, et al. Systematic review with meta-analysis of intraoperative neuromonitoring during thyroidectomy. Int J Surg 2017;39:104-13.

4. Randolph GW, Dralle H, Abdullah H, et al. Electrophysiologic recurrent laryngeal nerve monitoring during thyroid and parathyroid surgery: international standards guideline statement. Laryngoscope 2011;121 Suppl 1:S1-16.

5. Flisberg K, Lindholm T. Electrical stimulation of the human recurrent laryngeal nerve during thyroid operation. Acta Otolaryngol Suppl 1969;263:63-7.

6. Petro ML, Schweinfurth JM, Petro AB. Transcricothyroid, intraoperative monitoring of the vagus nerve. Arch Otolaryngol Head Neck Surg 2006;132:624-8.

7. Alon EE, Hinni ML. Transcricothyroid electromyographic monitoring of the recurrent laryngeal nerve. Laryngoscope 2009;119:1918-21.

8. Sturgeon C, Sturgeon T, Angelos P. Neuromonitoring in thyroid surgery: attitudes, usage patterns, and predictors of use among endocrine surgeons. World J Surg 2009;33:417-25.

9. Eisele DW. Intraoperative electrophysiologic monitoring of the recurrent laryngeal nerve. Laryngoscope 1996;106:443-9.

10. Lu IC, Chu KS, Tsai CJ, et al. Optimal depth of NIM EMG endotracheal tube for intraoperative neuromonitoring of the recurrent laryngeal nerve during thyroidectomy. World J Surg 2008;32:1935-9.

11. Tsai CJ, Tseng KY, Wang FY, et al. Electromyographic endotracheal tube placement during thyroid surgery in neuromonitoring of recurrent laryngeal nerve. Kaohsiung J Med Sci 2011;27:96-101.

12. Chambers KJ, Pearse A, Coveney J, et al. Respiratory variation predicts optimal endotracheal tube placement for intra-operative nerve monitoring in thyroid and parathyroid surgery. World J Surg 2015;39:393-9.

13. Wu CW, Chiang FY, Randolph GW, et al. Transcutaneous Recording During Intraoperative Neuromonitoring in Thyroid Surgery. Thyroid 2018;28:1500-7. 
14. Chiang FY, Lu IC, Chang PY, et al. Comparison of EMG signals recorded by surface electrodes on endotracheal tube and thyroid cartilage during monitored thyroidectomy. Kaohsiung J Med Sci 2017;33:503-9.

15. Zhao Y, Li C, Zhang D, et al. Experimental study of needle recording electrodes placed on the thyroid cartilage for neuromonitoring during thyroid surgery. Br J Surg 2019;106:245-54.

16. Wu CW, Chiang FY, Randolph GW, et al. Feasibility of Intraoperative Neuromonitoring During Thyroid Surgery Using Transcartilage Surface Recording Electrodes. Thyroid 2018;28:1508-16.

17. Liddy W, Lawson BR, Barber SR, et al. Anterior laryngeal electrodes for recurrent laryngeal nerve monitoring during thyroid and parathyroid surgery: New expanded options for neural monitoring. Laryngoscope 2018;128:2910-5.

18. Phelan E, Potenza A, Slough C, et al. Recurrent laryngeal nerve monitoring during thyroid surgery: normative vagal

Cite this article as: Li P, Liang QZ, Wang DL, Han B, Yi X, Wei W. Modified arytenoid muscle electrode recording method for neuromonitoring during thyroidectomy. Gland Surg 2019;8(5):469-476. doi: 10.21037/gs.2019.08.07 and recurrent laryngeal nerve electrophysiological data. Otolaryngol Head Neck Surg 2012;147:640-6.

19. Lorenz K, Sekulla C, Schelle J, et al. What are normal quantitative parameters of intraoperative neuromonitoring (IONM) in thyroid surgery? Langenbecks Arch Surg 2010;395:901-9.

20. Storck C, Gehrer R, Hofer M, et al. Laryngeal electromyography: electrode guidance based on 3-dimensional magnetic resonance tomography images of the larynx. J Voice 2012;26:110-6.

21. Dralle H, Sekulla C, Lorenz K, et al. Intraoperative monitoring of the recurrent laryngeal nerve in thyroid surgery. World J Surg 2008;32:1358-66.

22. Eid I, Miller FR, Rowan S, et al. The role of nerve monitoring to predict postoperative recurrent laryngeal nerve function in thyroid and parathyroid surgery. Laryngoscope 2013;123:2583-6. 